\title{
Die Intuitivitäts-Illusion: Intuitives Nutzererleben durch Attributionsfehler
}

\author{
Stefan Tretter ${ }^{1}$, Daniel Ullrich ${ }^{2}$, Sarah Diefenbach $^{1}$ \\ Department Psychologie, Ludwig-Maximilians-Universität München ${ }^{1}$ \\ Department Informatik, Ludwig-Maximilians-Universität München ${ }^{2}$
}

\section{Zusammenfassung}

Die sogenannte „Intuitivitäts-Illusion“ bezeichnet das Phänomen, dass Nutzer eine Produktinteraktion als intuitiv bewerten, obwohl sie tatsächlich ohne eine Instruktion im Vorfeld das Interaktionsziel nicht erreicht hätten. Ursache für dieses Phänomen sehen wir im Zusammenspiel von zwei Faktoren: OneShot-Learning und Hindsight-Bias. One-Shot-Learning bezeichnet die schnelle, beiläufige Vermittlung von Prinzipien (z.B. durch Beobachtungslernen). Dieses Wissen kann daraufhin zu einer erfolgreichen Interaktion beitragen. Durch den Rückschaufehler (Hindsight Bias) kommt es anschließend zu einer Fehlattribution. Anstatt auf die im Vorfeld gegebene Instruktion wird die erfolgreiche Interaktion auf das per se ,intuitive Produkt“ zurückgeführt. Der vorliegende Beitrag exploriert das Phänomen der Intuitivitäts-Illusion anhand einer Nutzerbefragung (N=80) zur Intuitivität von Touchgesten und diskutiert Implikationen für Design und zukünftige Forschung.

\section{Einleitung}

Bei der Interaktion mit technischen Produkten zählt nicht nur die einwandfreie Funktionalität, auch das Erleben des Nutzers während der Interaktion nimmt eine zentrale Rolle ein. Ein Teil dieses Erlebens beginnt mit der ersten Begegnung mit dem Produkt: Muss ich erst mühsam Anleitungen studieren oder vor jedem Mausklick überlegen, welcher Button wohl der richtige ist? Oder kann ich direkt loslegen, stets vom Gefühl geleitet, die richtige Funktion genau im passenden Moment zu finden und mühelos ans Ziel zu kommen? In letzterem Fall kann die Interaktion als ,intuitiv“ beschrieben werden.

Ob eine Interaktion auch wirklich als intuitiv empfunden wird, hängt von vielen Faktoren ab: Dem Vorwissen des Nutzers, dem Interaktionskontext und den Produkteigenschaften, um nur die wichtigsten zu nennen (Blackler \& Popovic 2015; Diefenbach \& Ullrich 2015). Am Ende steht der Nutzer, der aus den vielen Faktoren ein Gesamturteil integriert und die Interaktion als ,intuitiv“ bewertet - oder eben nicht. 


\section{Fallstricke der retrospektiven Bewertung}

Intuitivitätsurteile werden in der Regel retrospektiv und nach abgeschlossener Interaktion getroffen. Dadurch fließen eine Menge Aspekte in die Bewertung ein, die das Urteil verfälschen können. So werden beispielsweise einzelne Eindrücke überproportional stark gewichtet, wenn sie zu Beginn oder am Ende einer Interaktion stehen (Primacy- und RecencyEffekte; vgl. Ullrich 2011). Ebenso können besonders schlechte Einzelinteraktionen das ansonsten ,intuitive“ Gesamterleben zunichtemachen, was in globalen Urteilen nicht differenziert widergespiegelt wird. Hier schneidet die Interaktion mit einem Produkt dann insgesamt schlecht ab, obwohl nur eine einzelne Interaktion den Ausschlag gab (Ullrich 2011). Eine der Kernfragen im Kontext der intuitiven Interaktion ist es, welche Ursachen dafür verantwortlich sind, dass ein Nutzer ein Produkt intuitiv nutzen kann. Forscher im Feld der intuitiven Interaktion sind sich weitgehend einig, dass im Vorfeld angeeignetes Wissen bei der Interaktion unbewusst zur Anwendung kommt (Blackler \& Popovic 2015; Fischer et al. 2015; Diefenbach \& Ullrich 2015). Genau diese unbewusste Anwendung macht das Phänomen so schwer greifbar und führt zu (letztlich wenig aussagekräftigen) Begründungen wie „natürlich bedient man es so“.

Die rückblickende Natur der Bewertung im Zusammenspiel mit Prozessen, die sich der bewussten Wahrnehmung entziehen, macht die Produktbewertung dabei besonders fehleranfällig. So besagt das gut fundierte Phänomen des „Rückschaufehlers“ (Hindsight Bias; Christensen-Szalanski \& Willham 1991), dass Menschen im Anschluss an den Ausgang eines Ereignisses systematischen Verzerrungen unterliegen, was ihren Wissensstand vor Eintritt des Ereignisses betrifft. Wir nehmen an, dass dieses Phänomen auch im Zusammenhang mit intuitiver Interaktion eine Rolle spielt, was als „Intuitivitäts-Illusion“ bezeichnet werden kann.

Diese Illusion ließe sich in der Produktgestaltung zunutze machen. Im Zentrum unserer Forschung steht daher die Frage, ob es möglich ist, Nutzern das für die Interaktion notwendige Wissen - bewusst oder unbewusst - zukommen zu lassen und die Nutzer die in der Folge erfolgreiche und als intuitiv wahrgenommene Interaktion retrospektiv auf das Produkt attribuieren. Forschungsergebnisse können für das Design interaktiver Produkte und begleitender Instruktionen (z.B. Manuals, Tutorials) wertvolle Einsichten liefern.

\section{Nutzerbefragung: Erste Einsichten}

In einer explorativen Studie haben wir 80 Personen mittels Paper-Pencil-Fragebogen zu ihrer ersten Erfahrung mit der Nutzung eines Smartphones (mit Touchscreen) befragt, insbesondere zur Beurteilung der Intuitivität der Zoom-Geste (zwei Finger, auseinanderziehen). Die Zoom-Geste sahen wir als ein interessantes Forschungsbeispiel, da diese eine der populärsten Touch-Gesten darstellt und allein durch ihre Bekanntheit mittlerweile als „intuitiv“ gelten könnte. 77 Personen (60 weiblich; Durchschnittsalter $M=22,97$, Min=19, Max=65) haben die Fragen komplett beantwortet und bilden die Datenbasis für die Auswertung. Die Teilnehmer 
konnten sich im Mittel sehr gut an ihre erste Nutzung eines Smartphones erinnern $(M=5,29$; $S D=1,87$; Skala 1-7) und es war ihnen direkt klar, wie sie das Gerät bedienen mussten $(M=5,19 ; S D=1,67$; Skala 1-7). Die Zoom-Geste wurde von den Nutzern als sehr intuitiv bewertet $(M=6,09 ; S D=1,22$; Skala 1-7). Die Nutzer gaben zudem an, dass ihnen sofort klar war, wie die Zoom-Geste zu verwenden ist $(M=6,08 ; S D=1,16)$. Das Prädikat ,intuitiv“ ist der Zoom-Geste also sicher. Eine interessante Frage ist, auf welcher Basis dieser Eindruck entsteht und welche Art von Vorwissen die erfolgreiche Interaktion ermöglicht. Auf die Frage, woher sie wussten, wie die Zoom-Geste funktioniert, gaben 52\% der Teilnehmer an, die Geste sei intuitiv und unmittelbar einleuchtend gewesen oder sie hätten es durch Ausprobieren selbst herausgefunden. Lediglich 27,3\% der Befragten antworteten, sie hätten die Bedienung von jemandem gezeigt bekommen oder in der Werbung gesehen. Die übrigen Teilenehmer gaben an, sich nicht mehr erinnern zu können.

Offenbar wird die Zoom-Geste von den meisten Nutzern rückblickend als intuitiv bewertet. Gleichzeitig berichtet die Mehrheit der Nutzer, sie sei selbst auf die Funktion gekommen oder wisse es schlicht nicht mehr. Beides steht im Einklang mit dem Konzept der intuitiven Interaktion, bei dem es gerade um die unbewusste Anwendung von Vorwissen geht, die sich naturgemäß der Verbalisierung einer Begründung entzieht (Diefenbach \& Ullrich 2015). Eine Teilnehmerrückfrage lautete bezeichnend: „Kann man auch mehrfach ankreuzen? Ich weiß, dass es mir in der Werbung gezeigt wurde, aber die Geste ist einfach auch sehr intuitiv." Aussagen wie diese zeigen deutlich, dass eine vorherige Lernerfahrung in den Hintergrund tritt und eine Verzerrung in der rückblickenden Einschätzung einer Interaktion eine „Illusion“ von Intuitivität erzeugt, die es ohne die Lernerfahrung wohl nicht gegeben hätte.

\section{Diskussion}

Ein Grund für die Eingängigkeit der Touch-Bedienung im Allgemeinen ist die Tatsache, dass sie das Interaktionskonzept der haptischen Berührung und Manipulation (z.B. Berühren und Verschieben eines Stück Papiers) in einem technischen Kontext abbildet (Chattopadhyay \& Bolchini 2015). Funktionen wie Drag\&Drop oder Umblättern wurden bereits in der ,,wirklichen Welt" gelernt und das Konzept muss nur noch im Kontext Smartphone angewandt werden, was einen kleinen Schritt darstellt, sobald die Metapher verstanden ist (vgl. OneShot-Learning; Lee 2015).

Bei der Zoom-Geste verhält es sich anders, da es hier kein Äquivalent in der realen Welt gibt. Buchstaben auf einem Blatt oder Fotos werden nicht größer, wenn man sie mit zwei Fingern streckt. Es ist daher nicht naheliegend, dass Nutzer ,,von sich aus“ darauf kommen diese unbekannte Geste in einem neuen Kontext zu nutzen. Unserer Einschätzung nach liegt hier der Fall eines Rückschaufehlers vor: Die Nutzer haben die Interaktion im Vorfeld bereits gesehen - sei es bei Freunden oder in einem von zahlreichen Werbespots. Bei der konkreten Interaktion ist das Konzept somit nicht mehr gänzlich unbekannt und die Hürde, es selbst anzuwenden, entsprechend geringer. Gleichzeitig ist die Quelle des Wissens (Freunde, Werbung) aber nicht mehr präsent, sodass auf das Produkt attribuiert wird. Eine Instruktion kann hierbei von einer bewussten Anleitung bis zu beiläufigem Beobachtungslernen reichen. 


\section{Ausblick}

Zukünftige Studien sollen untersuchen, welche Form der Instruktion mit welchen Arten des Vorwissens optimal kombinierbar ist und welche Kombinationen zu den besten Resultaten führen. Hierfür planen wir eine experimentelle Studie, in der das Maß der im Vorfeld gegebenen Information variiert wird. Teilnehmer bekommen (a) keine Anleitung, (b) eine Anleitung per Manual oder (c) eine Anleitung per Video und müssen anschließend mit einem ihnen unbekannten Programm arbeiten, das für bestimmte Funktionen Maus-Gesten vorsieht. Gemessen werden Effektivitätsmaße, die subjektive Intuitivität und woher die Teilnehmer wussten, wie die Sonderfunktionen zu nutzen sind. Falls die Nutzer nach erfolgreicher (instruierter) Interaktion die Intuitivität auf das Produkt attribuieren, würden wir dies als starkes Indiz für unser Konzept der induzierten Intuitivität sehen. Gleichzeitig würde diese Erkenntnis tieferen Einblick in die unbewussten Prozesse bieten, die dem Intuitivitätserleben eines Nutzers zugrunde liegen. Weiterführend stellte sich die Frage, wie Produktdesigner es schaffen können, Interaktionsinstruktionen bzw. entsprechende Cues in das Produkt selbst zu integrieren, um es unabhängig von vorherigen Lernerfahrungen (z.B. Marketingkampagnen) zu machen. Neben der Aktivierung von Vorwissen wäre somit das beiläufige Schaffen von Wissen eine zusätzliche Herausforderung, um intuitive Interaktion zu ermöglichen.

\section{Literaturverzeichnis}

Blackler, A., \& Popovic, V. (2015). Towards intuitive interaction theory. Interacting with Computers, 27(3), 203-209.

Chattopadhyay, D., \& Bolchini, D. (2015). Motor-intuitive interactions based on image schemas: Aligning touchless interaction primitives with human sensorimotor abilities. Interacting with Computers, 27(3), 327-343.

Christensen-Szalanski, J. J., \& Willham, C. F. (1991). The hindsight bias: A meta-analysis. Organizational Behavior and Human Decision Processes, 48(1), 147-168.

Diefenbach, S., \& Ullrich, D. (2015). An experience perspective on intuitive interaction: Central components and the special effect of domain transfer distance. Interacting with Computers, 27(3), 210234 .

Fischer, S., Itoh, M., \& Inagaki, T. (2015). Screening prototype features in terms of intuitive use: Design considerations and proof of concept. Interacting with Computers, 27(3), 256-270.

Lee S.W., O’Doherty J.P., \& Shimojo S. (2015). Neural computations mediating one-shot learning in the human brain. PLOS Biology, 13(4), e1002137.

Ullrich, D. (2011). Primacy- und Recency-Effekt bei der Produktinteraktion und ihr Einfluss auf die Bewertung von Inuitivität und User Experience. In: Proceedings 7.Tagung der Fachgruppe Arbeits-, Organisations- und Wirtschaftspsychologie, 124-125.

\section{Kontaktinformationen}

Stefan Tretter, Ludwig-Maximilians-Universität München, stefan.tretter@psy.lmu.de 